\title{
Synthesis of New 2,3-Dihydro-2-phenyl-4-quinolone Derivatives; Aza Analogs of Flavanone
}

\author{
Myung-Sook Park" and Jae-ln Lee \\ College of Phamacy. Duksung Women's liniversity, Seoul 132-7/4. Korea \\ ${ }^{\dagger}$ Department of Chemistry; Duksung Women's Liniversity, Seoul 132-714. Korea \\ Received Mo: 2, 2004
}

Key Words : 2,3-Dihydro-2-phenyl-4-quinolones, Synthesis, $\Lambda$ ntiinflammatory, $\Lambda z a$ analogs of flavanone

Flavonoids are natural polyphenol compounds of plant origin and exhibit various biological activities such as antiinflammatory, anti-oxidant, and anti-tumor activities. ${ }^{1-\overline{3}}$ It has been previously reported that various plant flavonoids possess the inhibitory activity on cyclooxygenase/lipoxygenase. ${ }^{\text {th }}$

Based on the results from the structure-activity relationships of Hesperetin and some other naturally occurring flavanone such as Taxifolin (Figure 2) and in order to improve the antiinflammatory activities of Hesperetin, we<smiles>[R][R]1ccc(C2CC(=O)c3ccccc3O2)cc1</smiles>

Flavanones<smiles>[R]c1ccc(C2CC(=O)c3ccccc3N2)cc1</smiles>

2,3-Dihydro-2-phenylquinolones
Figure 1. Siruclure of llavonones. designed aza analogs of flavanones that were replaced the oxygen atom of $B$ ring of flavanone to the nitrogen atom (Figure 1). In this work, we report the synthesis of the title compound 3, novel 2,3-dihydro-2-phenyl-4-cjuinolone derivatives as a potential candidate for NSAIDs (nonsteriodal antiinflammatory drugs).

A series of 2,3-dihydro-2-phenyl-4-quinolones 3a-g has been synthesized using acid-catalyzed one-pot reaction (Scheme 1), Quinolones 3a-g were prepared through cyclization of the condensation product $2 \mathbf{a}-\mathbf{g}$ that were formed by heating of arylamines and ethyl benzoylacetate in toluene. Similarly, the 6 (7 or 8)-substituted 2,3-dihydro-2phenylquinolones were prepared from the paralortho or meta)-substituted aniline. The reaction mechanism of the formation of the final product involves the nucleophilic dehydration and following cyclization between arylamines and ethyl benzoylacetate (Г:BA). Nucleophilic dehydration. the condensation was undertaken with $p$-toluene sulfonic acid at $90-110^{\circ} \mathrm{C}$ in toluene for $2-6$ hours over the dean-<smiles>O=C1c2c(O)cc(O)cc2OC(c2ccc(O)c(O)c2)C1O</smiles>

Taxifolin<smiles>COc1ccc(C2CC(=O)c3c(O)cc(O)cc3O2)cc1O</smiles>

Hesperetin<smiles>COc1cc(O)c2c(c1)OC(c1ccc(OC)c(O)c1)CC2=O</smiles>

Hesperidin
Figure 2. Structure of tlavanones with antiinllammatory activity.

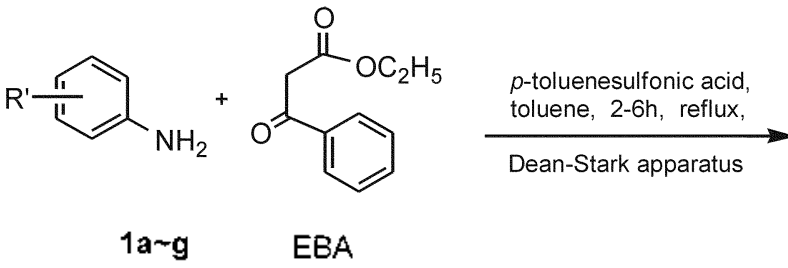<smiles>[R]OC(=O)/C=C(/Nc1ccccc1)c1ccccc1</smiles><smiles>[R]c1ccc2c(c1)NC(c1ccccc1)CC2=O</smiles>

$3 a \sim g$

$\begin{array}{lllc} & \mathbf{R}^{\prime} & & \mathbf{R}^{\prime} \\ \text { 1a, 2a } & \mathrm{H} & \text { 1e, 2e } & o-\mathrm{CH}_{3} \mathrm{O} \\ \text { 1c, 2c } & m-\mathrm{CH}_{3} \mathrm{CO} & 1 \mathrm{f}, 2 \mathrm{f} & m-\mathrm{CH}_{3} \mathrm{O} \\ \text { 1d, 2d } & p-\mathrm{CH}_{3} \mathrm{CO} & 1 \mathrm{~g}, \mathbf{2 g} & \rho-\mathrm{CH}_{3} \mathrm{O}\end{array}$

Scheme 1. Synthesis of 2,3-1)jhydro-2-phenylyuinolone I)erivatives 3a-g and Isolation of the inlemediate 2a-g.

Conesponding Aulher. e-mail: mspark ädduksung.ac.kr 


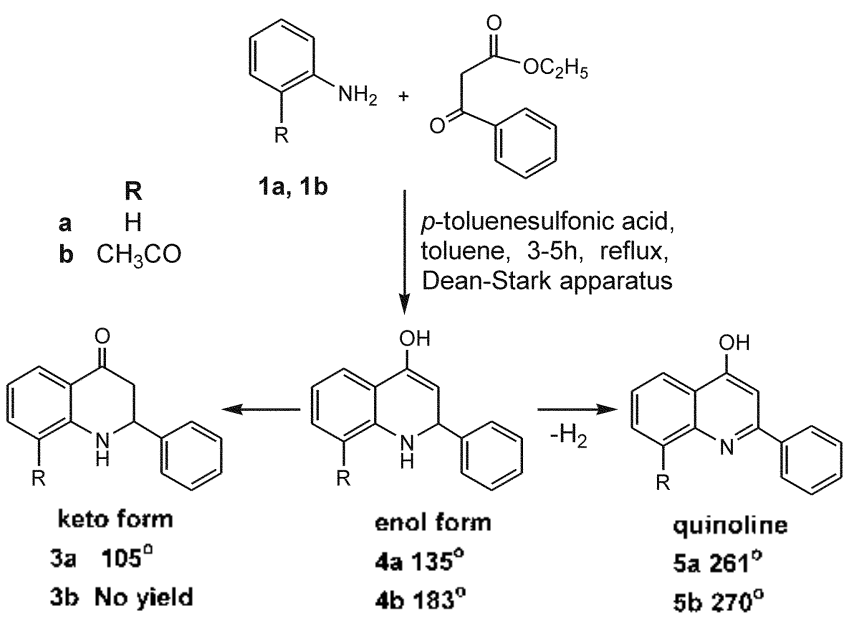

Scheme 2. Tautomerism of 2.3-Dihydro-2-phenylutuinolone Derivatives 3a and Dehydrogenation of $\mathbf{4 b}$.

stark apparatus for the ester 2 . The ester $\mathbf{2}$ was continuously converted to the 2-phenylquinolones $\mathbf{3}$ with removal of ethanol and dihydrogenation. All synthetic process from arylamines and ethyl benzoylacetate to quinolones $\mathbf{3 a - g}$ could be carried out in one-pot without isolation of intermediates.

Even though synthetic pathways for 2-phenyl-4-quinolones were developed by Li et al.. ${ }^{12}$ Kuo et al., ${ }^{13}$ Watterson et al..$^{14}$ and Xia et $a l . .^{15}$ the synthesis of 2,3-dihydro-2-phenyl4-quinolones has not been reported until now. We applied the synthetic method of one-pot operations for dihydroquinolone by Cho et al. ${ }^{10}$ We synthesized the 2.3-dihydro-2phenyl-4-quinolones using the reaction between ethyl benzoylacetate and arylamines.

The moiety that were introduced to aromatic nucleus of quinolones 3a-g were nonsubstituted, 7-acetyl, 6-acetyl, 8methoxy, 7-methoxy and 6-methoxy. Even though we efforted to complete cyclization in several methods, $\mathbf{2 a - g}$ were always isolated in reaction mixture. In the acid-catalytic dehydration, ethyl benzoylacetate was protonated and followed to transfer of electron. A molecule of water eliminated from nucleophillic attraction of aniline, C-N bond was formed for esters $\mathbf{2 a - g}$. Starting material nearly disappeared after about I hour after starting of reaction.

Even though esters 2a-g were converted to $\mathbf{3}$ and 4, they were left in reaction mixture in state of chemical equibrium (Scheme 2). Prolonged reaction time could not make into a complection of reaction, but resulted in a complicated reaction mixture. For the additional cyclization, ester $\mathbf{2} \mathbf{a}-\mathbf{g}$ were protonated at 2 and 3-position of ring, converted with 3 and 4 by eliminating ethanol.

Although enols $\mathbf{4 a}, \mathbf{b}$ were isolated and charaterized, we found that $4 \mathbf{a}$ was transformed to quinolone $3 \mathbf{a}$ in nonpolar solvent such as $\mathrm{CDCl}_{3}$. Enol 4 a was stable in state of solid, but changed to keto-form 3a as hydrogen transfer in solvent. Enol-form was transformed to more stable keto-form in the course of time. In the synthesis for $\mathbf{3} \mathbf{b}$, the product was not keto-form $\mathbf{3 b}$ but enol-form $\mathbf{4 b}$. Enol $\mathbf{4 b}$ was not transformed into enol $\mathbf{3 b}$ in solvent, but dehydrogenation at 1,2-position of quinolone ring took place during the process of reaction and chromatographic $\left(\mathrm{SiO}_{2}\right)$ isolation gave quinoline compound 5a-b (Scheme 2).

Enols $4 \mathbf{a}$, b and keto-form $3 \mathbf{a}$ were defined by ${ }^{1} \mathrm{H},{ }^{13} \mathrm{C}$ NMR, IR spectra and GC-MS. In the ${ }^{1} \mathrm{H}-\mathrm{NMR}, 11.02 \mathrm{ppm}$ of $\mathrm{OH}$ peak of enol-form was disappeared in keto-form. In ${ }^{1.3} \mathrm{C}-\mathrm{NMR}$ spectra of the enol- and keto-form, $\mathrm{C}-\mathrm{O}$ peak of enol-form appeared at $168.20 \mathrm{ppm}$, while $\mathrm{C}=\mathrm{O}$ peak of ketoform showed at the $196.50 \mathrm{ppm}$. Even though the carbonyl band of keto-form was clearly shown at about $1689 \mathrm{~cm}^{-1}$ on IR spectra, was not shown in case of enol-form.

Enol-form $\mathbf{4 b}$ was not transformed into keto-form $\mathbf{3 b}$ but into quinoline $\mathbf{5 b}$ because of a strong steric hindrance between 8-acetoxy moiety and $\mathrm{NH}$ of $\mathrm{B}$ ring and a weak conjugation effect. Keto-forms 3c (6-position) and 3d (7position) were produced in this condition since they do not have steric hindrance. The 8 -methoxy compound $3 \mathbf{e}$ was synthesized on less steric hindrance with $\mathrm{NH}$ of $\mathrm{B}$ ring because methoxy moiety was less bulky than acetoxy moiety. The reason for conversion of unsubstituted compound $\mathbf{4 a}$ into $3 \mathbf{a}$ in solvent such as $\mathrm{CDCl}_{3}$ is that keto-form is more stable than enol-form. Also, a part of $4 \mathbf{a}$ was transformed into quinoline 5 a due to conjugation effect. We thought that keto-form was more stable than enol-form because most of reaction was processed to yield keto-form $3 c-g$. We could not find enol-form in reaction of methoxy compound because electron donating methoxy group facilitate cyclization and additional transfer of proton to make keto-form.

Identification of products $\mathbf{2 a - g}$ and $\mathbf{3 a - g}$ were defined by spectra of NMR, IR and GC-MS. The NH peak of $\mathbf{3 a - g}$ was appeared at $9.2 \mathrm{ppm}$, a coupling of $\mathrm{CH}$ peak at $7.12 \mathrm{ppm}$ and $\mathrm{CH}_{2}$ peak at 4.1 I ppin was observed.

Finally, 2,3-dihydro-2-phenyl-4-quinolones 3a, 3c-g were synthesized through the condensation and cyclization of related amines and ethyl benzoylacetate. Esters 2 a-g were isolated as intermediates through one-pot reaction (Scheme 1).

\section{Experimental Section}

Chemicals were supplied by Aldrich, Sigma, Merck, and Tokyo Kasei. Melting points were determined in open capillary tubes on a Büchi 535 melting point apparatus and uncorrected. The NMR spectra were recorded using Bruker $300 \mathrm{MHz}$ NMR spectrometer. Chemical shift values were reported in parts per million on the scale in deuteriochloroform or dimethyl- $\mathrm{d}_{6}$ sulfoxide with tetramethylsilane as the internal standard. The NMR spin multiplicities were indicated by the symbols: $s$ (singlet), $d$ (doublet), $t$ (triplet), $q$ (quartet) and in (multiplet). IR spectra were recorded on a Petkin-Elmer l6F PC FT-IR spectrometer using $\mathrm{NaCl}$ discs and pellets. The Mass fragmentations were recorded using Agilent $6890 \mathrm{GC}$ and $5973 \mathrm{MS}$.

Isolation of intermediate 2a-g; 3-phenyl-3-anilinopropenoic acid ethyl ester (2a). mp $72.5^{\circ} \mathrm{C}$ TLC [ $n$-hexane : ethyl acetate (3:1)] Rf 0.73. 'H NMR (CDCl $) \delta 10.30$ (s, $1 \mathrm{H}, \mathrm{NH}), 7.35-7.24(\mathrm{~m}, 5 \mathrm{H}$, aromatic $), 7.08(\mathrm{t} . J=7.5 \mathrm{~Hz}$, 
$2 \mathrm{H}$, aromatic). $6.9 \mathrm{l}(\mathrm{t}, J=7.4 \mathrm{~Hz} . \mathrm{lH}$. aromatic), $6.65(\mathrm{~d} . J=$ $7.6 \mathrm{~Hz}, 2 \mathrm{H}$, aromatic), $4.99(\mathrm{~s}, \mathrm{lH} . \mathrm{CH}), 4.20$ (q. $J=7.1 \mathrm{~Hz}$. $\left.2 \mathrm{H}, \mathrm{CH}_{2}\right) .1 .31\left(\mathrm{t}, J=7.1 \mathrm{~Hz}, 3 \mathrm{H}, \mathrm{CH}_{3}\right) .{ }^{13} \mathrm{C} \mathrm{NMR}\left(\mathrm{CDCl}_{3}\right) \delta$ 170.10 (COO). 159.04. 140.35. 135.95. 129.42. 128.59. 128.41. 128.20, $122.17,91.14(\mathrm{CH}), 59.30\left(\mathrm{CH}_{2}\right), 14.52$ $\left(\mathrm{CH}_{3}\right) . \mathrm{IR}\left(\mathrm{NaCl} \mathrm{cm}^{-1}\right) 3259(\mathrm{NH}), 2979$ (aromatic). 1657 (CO).

3-Phenyl-3-(3-acetoxyanilino)propenoic acid ethyl ester (2c), oil TLC [n-hexane : ethyl acetate $(3: 2)] \mathrm{Rf} 0.57 .{ }^{1} \mathrm{H}$ NMR $\left(\mathrm{CDCl}_{3}\right) \delta 10.40$ (s, $\left.1 \mathrm{H}, \mathrm{NH}\right) .7 .56$ (s. $1 \mathrm{H}$. aromatic). $7.32(\mathrm{~m} .5 \mathrm{H}$, aromatic), 7.20 (s. $1 \mathrm{H}$, aromatic), 7.13 (t. $1 \mathrm{H}$. aromatic), 6.81 (d. $J=7.4 \mathrm{~Hz}, 2 \mathrm{H}$, aromatic), 5.05 (s. $1 \mathrm{H}$. $\mathrm{CH}$ ). 4.20 (q, $J=7.1 \mathrm{~Hz} .2 \mathrm{H}, \mathrm{CH}_{2}$ ), 3.32 (s, $2 \mathrm{H}, \mathrm{COCH}_{3}$ ). $1.30\left(\mathrm{t}, J=7.1 \mathrm{~Hz}, 3 \mathrm{H} . \mathrm{CH}_{3}\right) \cdot{ }^{13} \mathrm{C}$ NMR $\left(\mathrm{CDCl}_{3}\right) \delta 196.79$ $(\mathrm{C}=\mathrm{O}), 170.33(\mathrm{COO}) .158 .77,141.19 .137 .88,131.60$. $130.07 .129 .26,129.13,129.04,128.90,126.47,122.77$. 122.02. $92.80(\mathrm{CH}), 60.68\left(\mathrm{CH}_{2}\right), 26.79\left(\mathrm{CH}_{3}\right) 1+.58\left(\mathrm{CH}_{3}\right)$. IR (pellet, $\mathrm{cm}^{-1}$ ) $3367(\mathrm{NH}), 2980$ (aromatic), $1740(\mathrm{CO})$. $1664(\mathrm{CO})$.

3-Phenyl-3-(4-acetoxyanilino)propenoic acid ethyl ester (2d). oil TLC [n-hexane : ethyl acetate $(3: 1)] \mathrm{Rf} 0.44 .{ }^{~} \mathrm{H}$ $\operatorname{NMR}\left(\mathrm{CDCl}_{3}\right) \delta 10.73(\mathrm{~s}, 1 \mathrm{H}, \mathrm{NH}) .7 .68(\mathrm{~d}, J=7.4 \mathrm{~Hz} .2 \mathrm{H}$. aromatic), 7.34 (m. $5 \mathrm{H}$, aromatic), 6.64 (d. $J=7.4 \mathrm{~Hz} .2 \mathrm{H}$. aromatic), $5.11(\mathrm{~s}, 1 \mathrm{H}, \mathrm{CH}) .4 .23\left(\mathrm{q} . J=7.1 \mathrm{~Hz} .2 \mathrm{H} . \mathrm{CH}_{2}\right.$ ). $2.46\left(\mathrm{~s}, 2 \mathrm{H} . \mathrm{COCH}_{3}\right), 1.30\left(\mathrm{t}, J=7.1 \mathrm{~Hz} .3 \mathrm{H} . \mathrm{CH}_{3}\right) \cdot{ }^{13} \mathrm{C}$ NMR $\left(\mathrm{CDCl}_{3}\right) \delta 196.69(\mathrm{C}=\mathrm{O}), 169.77(\mathrm{COO}) .155 .44$. $144.98 .133 .73,129.80,129.39,128.77,128.49,120.24$. $94.28(\mathrm{CH}), 61.37\left(\mathrm{CH}_{2}\right) .29 .28\left(\mathrm{CH}_{3}\right) 14.44\left(\mathrm{CH}_{3}\right) . \mathrm{IR}$ (pellet. $\mathrm{cm}^{-1}$ ) $3367(\mathrm{NH}), 2980$ (aromatic). 1740 (CO). 1664 (CO).

3-Phenyl-3-(2-methoxyanilino)propenoic acid ethyl ester (2e). mp $105^{\circ} \mathrm{C}$ TLC [ $n$-hexane : ethyl acetate $\left.(2: 1)\right] \mathrm{Rf}$ 0.63. ${ }^{1} \mathrm{H}$ NMR $\left(\mathrm{CDCl}_{3}\right) \delta 10.27$ (s. IH. NH), 7.36-7.27 (m. $5 \mathrm{H}$, aromatic). 6.83 (n, $2 \mathrm{H}$. aromatic), $6.49(\mathrm{t}, J=7.2 \mathrm{~Hz}$. $1 \mathrm{H}$, aromatic), 6.20 (d. $J=7.2 \mathrm{~Hz}, 1 \mathrm{H}$, aromatic). 4.99 (s. $1 \mathrm{H}, \mathrm{CH}), 4.21\left(\mathrm{q}, J=7.2 \mathrm{~Hz} .2 \mathrm{H}, \mathrm{CH}_{2}\right), 3.88\left(\mathrm{~s}, 3 \mathrm{H} . \mathrm{OCH}_{3}\right)$. $1.30\left(\mathrm{t}, J=7.2 \mathrm{~Hz}, 3 \mathrm{H} . \mathrm{CH}_{3}\right) \cdot{ }^{13} \mathrm{C}$ NMR $\left(\mathrm{CDCl}_{3}\right) \delta 170.25$ (COO). 158.79, 150.89. 136.78, 130.01, 129.78, 128.79 . 128.39. 123.29, 122.19, 120.27. 110.91, $91.99(\mathrm{CH}) .59 .65$ $\left(\mathrm{OCH}_{3}\right) .56 .11\left(\mathrm{OCH}_{3}\right) 14.98\left(\mathrm{CH}_{3}\right)$.

3-Phenyl-3-(3-methoxyanilino)propenoic acid ethyl ester (2f). oil TLC [n-hexane : ethyl acetate $(2: 1)] \mathrm{Rf} 0.59 .{ }^{~} \mathrm{H}$ NMR $\left(\mathrm{CDCl}_{3}\right) \delta 10.29$ (s. $\left.1 \mathrm{H} . \mathrm{NH}\right), 7.37-7.28(\mathrm{~m} .5 \mathrm{H}$. aromatic). 6.96 (t. $J=8.1 \mathrm{~Hz} .1 \mathrm{H}$. aromatic). 6.26 (t. $J=8.1$ $\mathrm{Hz}, 1 \mathrm{H}$. aromatic), 6.16 (d. $J=8.1 \mathrm{~Hz}, 1 \mathrm{H}$, aromatic) 4.99 (s. $1 \mathrm{H}, \mathrm{CH}$ ). 4.20 (q. $J=7.2 \mathrm{~Hz}, 2 \mathrm{H}, \mathrm{CH}_{2}$ ). 3.53 (s. $3 \mathrm{H}$. $\left.\mathrm{OCH}_{3}\right) .1 .31$ (t. $\left.J=7.2 \mathrm{~Hz} .3 \mathrm{H} . \mathrm{CH}_{3}\right){ }^{13} \mathrm{C}$ NMR $\left(\mathrm{CDCl}_{3}\right) \delta$ 170.46 (COO). 160.14. 159.28. 141.95. 136.50. 129.85. 129.68, 128.86. 128.53, 114.82, 109.44, 107.83, $91.78(\mathrm{CH})$. $59.75\left(\mathrm{OCH}_{2}\right) .55 .37\left(\mathrm{OCH}_{3}\right) .14 .92\left(\mathrm{CH}_{3}\right)$

3-Phenyl-3-(4-methoxyanilino)propenoic acid ethyl ester (2g). mp $115^{\circ} \mathrm{C}$ TLC [ $n$-hexane : ethyl acetate $\left.(2: 1)\right] \mathrm{Rf}$ 0.6. ${ }^{1} \mathrm{H}$ NMR $\left(\mathrm{CDCl}_{\hat{j}}\right) \delta 10.22$ (s. 1H. NH) . 7.36-7.28 (m. $5 \mathrm{H}$ aromatic). $6.62(\mathrm{~m} .4 \mathrm{H}$ aromatic). 4.93 (s. $1 \mathrm{H} . \mathrm{CH})$. 4.21 (q. $\left.J=7.1 \mathrm{~Hz}, 2 \mathrm{H}, \mathrm{CH}_{2}\right) .3 .69$ (s. $3 \mathrm{H}, \mathrm{OCH}_{3}$ ). 1.33 (t. $J$ $\left.=7.1 \mathrm{~Hz} \cdot 3 \mathrm{H} . \mathrm{CH}_{3}\right) \cdot{ }^{13} \mathrm{C} \mathrm{NMR}\left(\mathrm{CDCl}_{3}\right) \delta 170.29(\mathrm{COO})$. 159.88. $155.78,136.01,133.46,129.22,128.34,128.28$.
124.24, 113.84. $89.51(\mathrm{CH}) .59 .16\left(\mathrm{OCH}_{2}\right), 55.30\left(\mathrm{OCH}_{3}\right)$ $14.55\left(\mathrm{CH}_{3}\right) . \mathrm{IR}\left(\mathrm{NaCl}, \mathrm{cm}^{-1}\right) 3255(\mathrm{NH}) .2976$ (aromatic), 1734 (CO). $1652(\mathrm{CO})$.

General procedure for the synthesis of 3a, 3c-g; 2,3Dihydro-2-phenyl-4-quinolone (3a). Ethyl benzoylacetate (1.72 mL. $0.01 \mathrm{~mol}$ ), $p$-toluenesulfonic acid (19.2 $\mathrm{mg} .0 .1$ mmol) and aniline $(1 \mathrm{~mL} .0 .011 \mathrm{~mol})$ were refluxed for about $4 \mathrm{~h}$ in anlyydrous toluene $(30 \mathrm{~mL}$ ) over the Dean-stark apparatus until the starting material disappeared. The reaction mixture was allowed to cool to room temperature, concentrated under reduced pressure to remove the toluene. The residue was extracted with ethyl acetate $(30 \mathrm{~mL})$ and water $(15 \mathrm{~mL})$ and the organic layer was washed with $5 \%$ $\mathrm{NaHCO}_{3}(15 \mathrm{~mL})$, water $(15 \mathrm{~mL} \times 2)$. The extracts were dried over anhydrous $\mathrm{Na}_{2} \mathrm{SO}_{4}$, and concentrated under a reduced pressure. The residue was purified by column chromatography to give compound $3 \mathrm{a}$ as a high viscous oil. The oil was crystallized from methanol.

Yield: $31.3 \%, \mathrm{mp} 105^{\circ} \mathrm{C}$ TLC [ $n$-hexane : ethyl acetate (3: 1)] Rf 0.27 . ${ }^{1} \mathrm{H} \mathrm{NMR}\left(\mathrm{CDCl}_{3}\right) \delta 9.29$ (s. $\left.\mathrm{lH}, \mathrm{NH}\right) .8 .04$ (d. $J=7.2 \mathrm{~Hz} .2 \mathrm{H}$, aromatic), 7.48 (m. $6 \mathrm{H}$, aromatic), 7.33 (t. $J=7.2 \mathrm{~Hz}, 2 \mathrm{H}$, aromatic), $7.12(\mathrm{t}, J=7.2 \mathrm{~Hz} .1 \mathrm{H} . \mathrm{CH}$ ), $4.11\left(\mathrm{~s}, 2 \mathrm{H}, \mathrm{CH}_{2}\right) .{ }^{12} \mathrm{C} \mathrm{NMR}\left(\mathrm{CDCl}_{3}\right) \delta 196.50(\mathrm{C}=\mathrm{O})$. $163.74,137.56$. 136.07, 134.37, 129.00. 128.62. 124.57, $120.20(\mathrm{CH}), 45.52\left(\mathrm{CH}_{2}\right)$. IR (pellet $\mathrm{cm}^{-1}$ ) $3307(\mathrm{NH}) .3063$ (aromatic). $1689(\mathrm{C}=\mathrm{O})$. GC.MS: $\mathrm{m} / \mathrm{z} 93.10(100.00), 66.10$ (30.79). $65.10(15.44), 92.20$ (12.31). $94.10(7.13)$.

7-Acetoxy-2,3-dihydro-2-phenyl-4-quinolone (3c). Yield: $43.9 \%, \mathrm{mp} 151{ }^{\circ} \mathrm{C}$ TLC $[\eta$-hexane : ethyl acetate $(3: 2)] \mathrm{Rf}$ $0.3 \mathrm{l} .{ }^{1} \mathrm{H}$ NMR $\left(\mathrm{CDCl}_{3}\right) \delta 9.60(\mathrm{~s}, \mathrm{lH} . \mathrm{NH}), 8.44(\mathrm{~m}, 2 \mathrm{H}$. aromatic). $8.10(\mathrm{~m} . \mathrm{HH}$. aromatic). $7.58(\mathrm{~m} .3 \mathrm{H}$, aromatic), 7.49 (m. $2 \mathrm{H} . \mathrm{CH}) .4 .12\left(\mathrm{~s}, 2 \mathrm{H} . \mathrm{CH}_{2}\right), 2.55\left(\mathrm{~s}, 3 \mathrm{H}, \mathrm{CH}_{3}\right) .{ }^{13} \mathrm{C}$ NMR $\left(\mathrm{CDCl}_{3}\right) \delta 198.89(\mathrm{C}=\mathrm{O}), 196.32(\mathrm{C}=\mathrm{O}) .165 .05$. 138.09, 137.78. 136.34, 134.72, 129.69. 129.34. 128.89. $125.20,124.71 .120 .16 .46 .25\left(\mathrm{CH}_{2}\right) .27 .46\left(\mathrm{CH}_{3}\right)$. IR (pellet $\left.\mathrm{cm}^{-1}\right) 3345(\mathrm{NH}) .1681,1656(\mathrm{C}=0)$. GC-MS: $\mathrm{m} / \mathrm{z} 222.20$ $(100.00), 237.20(54.07)$. 223.10 (17.30), 91.10 (12.96), $238.20(9.58)$.

6-Acetoxy-2,3-dihydro-2-phenyl-4-quinolone (3d). Yield: $67.4 \%$ mp $164^{\circ} \mathrm{C}$ TLC $[n$-hexane : ethyl acetate $(2: 1)] \mathrm{Rf}$ 0.21 . ${ }^{l} \mathrm{H}$ NMR $\left(\mathrm{CDCl}_{3}\right) \delta 9.69$ (s. $1 \mathrm{H} . \mathrm{NH}$ ). 8.03 (d. $J=6.9$ Hz. $2 \mathrm{H}$, aromatic). 7.99 (d. $J=6.9 \mathrm{~Hz}, 2 \mathrm{H}$, aromatic), 7.68 (m. $3 \mathrm{H}$. aromatic). 7.55 (t. $J=6.9 \mathrm{~Hz}, 2 \mathrm{H} . \mathrm{CH}) .4 .15$ (s. $2 \mathrm{H}$. $\left.\mathrm{CH}_{2}\right) .2 .55$ (s. $\left.3 \mathrm{H}_{2} \mathrm{CH}_{3}\right){ }^{13} \mathrm{C} \mathrm{NMR}\left(\mathrm{CDCl}_{3}\right) \delta 197.01(\mathrm{C}=\mathrm{O})$. $196.42(\mathrm{C}=0), 164.08,141.88,135.87,134.60,133.13$. $129.73,129.06,128.60,119.38,45.20\left(\mathrm{CH}_{3}\right), 26.46\left(\mathrm{CH}_{3}\right)$. IR (pellet cm $\left.\mathrm{cm}^{-1}\right) 3435(\mathrm{NH}) .1687,1663(\mathrm{C}=\mathrm{O})$. GC-MS: $\mathrm{m} / \mathrm{z} 120.10$ (100.00). $135.10(51.28) .92 .10(37.80) .65 .10$ (21.62). $121.10(8.26)$.

8-Metoxy-2,3-dihydro-2-phenyl-4-quinolone (3e). Yield: $41.6 \%$ mp $87^{\circ} \mathrm{C}$ TLC [ $n$-hexane : ethyl acetate $\left.(2: 1)\right] \mathrm{Rf}$ 0.37 . ${ }^{1} \mathrm{H}$ NMR $\left(\mathrm{CDCl}_{3}\right) \delta 9.40$ (s. lH, NH), 8.37 (d. $J=8.1$ Hz. $1 \mathrm{H}$. aromatic), 7.96 (d. $2 \mathrm{H}$. aromatic) $7.43-7.36(\mathrm{~m} .3 \mathrm{H}$. aromatic). $7.01-6.80\left(\mathrm{~m} .3 \mathrm{H}\right.$. aromatic). 4.11 (s. $\left.2 \mathrm{H} . \mathrm{CH}_{2}\right)$. 3.82 (s. $\left.3 \mathrm{H}, \mathrm{OCH}_{3}\right) .{ }^{13} \mathrm{C}$ NMR $\left(\mathrm{CDCl}_{3}\right) \delta 196.09(\mathrm{C}=\mathrm{O})$. 164.24 (CO). $148.76,136.53 .134 .52,134.44 .129 .24$ $129.00,128.88 .128 .35,127.90,126.23,124.51,121.40$. 
120.51. 110.55, $56.22\left(\mathrm{OCH}_{3}\right), 46.99\left(\mathrm{CH}_{2}\right) . \mathrm{IR}\left(\mathrm{NaCl} . \mathrm{cm}^{-1}\right)$ $3291(\mathrm{NH}) .3089$ (aromatic), $1693(\mathrm{C}=\mathrm{O})$. GC-MS: $\mathrm{m} / \mathrm{z}$ $108.10(100.00), 123.10(99.66), 80.10(70.35), 53.10$ (12.48), $52.10(8.69)$.

7-Metoxy-2,3-dihydro-2-phenyl-4-quinolone (3f). Yield: $55.3 \%$. oil TLC [ $n$-hexane : ethyl acetate $(2: 1)]$ Rf $0.32 .{ }^{~} \mathrm{H}$ NMR $\left(\mathrm{CDCl}_{3}\right) \delta 9.26$ (s. IH. NH). 7.94 (d. $J=7.5 \mathrm{~Hz}, 2 \mathrm{H}$. aromatic). 7.55 (t. $J=7.5 \mathrm{~Hz}$. IH. aromatic). 7.42 (t. $J=7.5$ $\mathrm{Hz}, 2 \mathrm{H}$. aromatic). $7.2 \mathrm{l}$ (s. $1 \mathrm{H}$, aromatic), 7.13 (t. $1 \mathrm{H}$. aromatic), 6.99 (d, $J=7.2 \mathrm{~Hz}, 1 \mathrm{H}$. aromatic), 6.60 (dd. $1 \mathrm{H}$. $\mathrm{CH}) .403\left(\mathrm{~d} . J=10.1 \mathrm{~Hz} .2 \mathrm{H}, \mathrm{CH}_{2}\right) .3 .7 \mathrm{l}\left(\mathrm{s}, 3 \mathrm{H}, \mathrm{OCH}_{3}\right) .{ }^{13} \mathrm{C}$ NMR $\left(\mathrm{CDCl}_{3}\right) \delta 196.75(\mathrm{C}=\mathrm{O}) .164 .33,160.48,139.16$. 136.38. 134.75, 130.08, 129.36, 129.00, 128.88, 128.45. 112.76. $110.86 .106 .20 .55 .70\left(\mathrm{OCH}_{3}\right), 46.02\left(\mathrm{CH}_{2}\right)$. GCMS: $\mathrm{m} / \mathrm{z} 73.10(100.00), 60.10(72.73), 55.10(59.93)$. $129.10(59.70), 57.10(57.74)$

6-Metoxy-2,3-dihydro-2-phenyl--t-quinolone (3g). Yield: $36.8 \%$. mp $120^{\circ} \mathrm{C}$ TLC [ $n$-hexane : ethyl acetate $(2: 1)$ ] Rf 0.26 . ${ }^{~} \mathrm{H}$ NMR $\left(\mathrm{CDCl}_{3}\right) \delta 9.24$ (s, lH. NH). 8.01 (d. $J=7.2$ $\mathrm{Hz}, 2 \mathrm{H}$. aromatic), $7.6 \mathrm{l}$ (m. $1 \mathrm{H}$, aromatic). 7.50 (t. $J=7.2$ $\mathrm{Hz}, 4 \mathrm{H}$, aromatic), 6.86 (d. $J=7.2 \mathrm{~Hz}, 2 \mathrm{H}$, aromatic), 4.09 (s. $\left.2 \mathrm{H}, \mathrm{CH}_{2}\right) .3 .78\left(\mathrm{~s}, 3 \mathrm{H} . \mathrm{OCH}_{3}\right),{ }^{13} \mathrm{C}$ NMR $\left(\mathrm{CDCl}_{3}\right) \delta$ $196.78(\mathrm{C}=\mathrm{O}) .164 .23,156.93 .136 .45 .134 .66,131.11$. 129.33. 128.98, 128.85, 122.41. 114.49, 55.84 $\left(\mathrm{OCH}_{3}\right)$. $45.89\left(\mathrm{CH}_{2}\right)$. IR $\left(\mathrm{NaCl}, \mathrm{cm}^{-1}\right) 3291(\mathrm{NH}), 3089$ (aromatic). $1693(\mathrm{C}=\mathrm{O})$. GC-MS: $\mathrm{m} / \mathrm{z} \mathrm{l} 08.10(100.00), 123.10(77.30)$. $80.10(33.06), 53.10(9.69), 109.10(7.39)$.

4-Hydroxy-2-hydro-2-phenyl-4-quinolone (4a). Yield: $45.5 \%$. mp $135.5^{\circ} \mathrm{C}$ TLC [ $n$-hexane : ethyl acetate $(3: 1)$ ] Rf 0.48. ${ }^{1} \mathrm{H} \mathrm{NMR}\left(\mathrm{CDCl}_{3}\right) \delta 11.02(\mathrm{~s}, \mathrm{lH} . \mathrm{OH}), 7.51(\mathrm{~d} . J=$ $7.5 \mathrm{~Hz}, 2 \mathrm{H}$. aromatic), $7.28(\mathrm{~m} .4 \mathrm{H}$, aronatic $+\mathrm{NH}), 7.03$ (m. $2 \mathrm{H}$, aromatic). $6.87(\mathrm{t}, J=7.5 \mathrm{~Hz} . \mathrm{H}$. aromatic), $6.62(\mathrm{~d}, J=$ $7.5 \mathrm{~Hz} .2 \mathrm{H}$, aromatic) 4.92 (s. $1 \mathrm{H}, \mathrm{CH}) .{ }^{13} \mathrm{C} \mathrm{NMR}\left(\mathrm{CDCl}_{3}\right) \delta$ 168.20 (C-O). 157.67, 140.51, 138.49. 136.36, 129.26. 128.94. 128.56, 128.51. 128.11, 122.57, $121.97(\mathrm{CH})$. 120.21. IR (pellet $\mathrm{cm}^{-1}$ ) 3307 (NH). 3058 (aromatic), 1592. GC-MS: m/z $180.10(100.00)$. $77.10(49.5), 195.10(47.99)$. $51.10(17.14), 181.10(14.01)$

8-Acetoxy-4-hydroxy-2-hydro-2-phenyl-4-quinolone (4b). Yield: $38.6 \% \mathrm{mp} 183{ }^{\circ} \mathrm{C}$ TLC [ $n$-hexane : ethyl acetate (1 : 1)] Rf 0.32. ${ }^{l} \mathrm{H} \mathrm{NMR}\left(\mathrm{CDCl}_{\mathfrak{s}}\right) \delta 8.50(\mathrm{~s} .1 \mathrm{H} . \mathrm{OH}) .8 .07$ (d. $J=7.2 \mathrm{~Hz}, 2 \mathrm{H}$. aromatic) $7.58(\mathrm{~m} .2 \mathrm{H}$. aromatic) 7.47 (m. $3 \mathrm{H}$. aromatic). $7.22(\mathrm{~m} .3 \mathrm{H}$. aromatic). 6.71 (d. $J=7.2$ $\mathrm{Hz} .1 \mathrm{H} . \mathrm{CH}$ ) 4.68 (s. $1 \mathrm{H} . \mathrm{CH}$ ) 1.63 (s. $3 \mathrm{H}, \mathrm{CH}_{3}$ ). ${ }^{13} \mathrm{C} \mathrm{NMR}$ $\left(\mathrm{CDCl}_{3}\right) \delta 197.04(\mathrm{C}=\mathrm{O}) .166 .63(\mathrm{C}-\mathrm{O}) .136 .75 .134 .20$. 130.14, 129.39, 128.94, 128.62. 124.36, 124.17, 115.87 . 72.48, 59.80, 27.38. IR (pellet $\mathrm{cm}^{-1}$ ) $3310(\mathrm{OH}) .3058$ (aromatic) 1705. 1646. 1595. GC-MS: m/z $120.10(100.00)$. $135.10(75.18), 92.10(42.89) .65 .10(25.20) .121 .10(8.13)$.

4-Hydroxy-2-phenylquinoline (5a). Yield: $16 \%$. mp 261 ${ }^{\circ} \mathrm{C}$ TLC [n-hexane : ethyl acetate $\left.(3: 1)\right]$ Rf 0.1. ${ }^{1} \mathrm{H}$ NMR (DMSO-d $) \delta 11.75$ (s. 1H. OH). 8.14 (d. $J=7.8 \mathrm{~Hz}, 1 \mathrm{H}$. aromatic). $7.84(\mathrm{~m} .3 \mathrm{H}$ aromatic). $7.80(\mathrm{~m} .1 \mathrm{H}$. aromatic). 7.69 (m. $3 \mathrm{H}$. aromatic). 7.22 (t. $J=7.8 \mathrm{~Hz} .1 \mathrm{H}$. aromatic) 6.39 (s. $1 \mathrm{H}, \mathrm{CH}$ ) ${ }^{13} \mathrm{C}$ NMR (DMSO-d 6$) \delta 177.75(\mathrm{C}-\mathrm{O})$.
$150.31,140.63 .134 .49,131.53,130.23,128.79 .127 .37$, $124.98,124.84 .123 .13,118.72,107.65(\mathrm{CH})$.

8-Acetoxy-4-hydroxy-2-phenylquinoline (5b). Yield: $28 \%$. mp $270{ }^{\circ} \mathrm{C}$ TLC [ 7 -hexane : etlyl acetate $\left.(1: 1)\right] \mathrm{Rf}$ 0.23 . ${ }^{1} \mathrm{H}$ NMR (DMSO-d) $\delta 12.02(\mathrm{~s} .1 \mathrm{H}, \mathrm{OH}), 7.87$ (dd, $J$ $=7.5 \mathrm{~Hz}, 3 \mathrm{H}$, aromatic). $7.66(\mathrm{t} .1 \mathrm{H}$, aromatic) $.7 .52(\mathrm{~m} .3 \mathrm{H}$. aromatic $+\mathrm{CH}) .7 .40(\mathrm{~d} . J=7.5 \mathrm{~Hz}, \mathrm{lH}$, aromatic). $7.26(\mathrm{t}, J$ $=7.5 \mathrm{~Hz}, \mathrm{lH}$, aromatic) $2.28\left(\mathrm{~s} .3 \mathrm{H} . \mathrm{CH}_{3}\right) \cdot{ }^{12} \mathrm{C} \mathrm{NMR}$ (DMSO-d $\left.\mathrm{d}_{6}\right) 195.50(\mathrm{C}=0) .159 .7 \mathrm{l}(\mathrm{C}-\mathrm{O}), 144.39,138.28$. 136.41, 133.73. 130.83, 130.80, 128.81. 125.07. 122.10, 119.10, 115.72 (CH), 15.57. IR (pellet $\left.\mathrm{cm}^{-1}\right) 3449(\mathrm{OH})$. 2993 (aromatic), $1687(\mathrm{C}=\mathrm{O}) .1640(\mathrm{C}-\mathrm{O})$.

Acknowledgment. This work was supported by the grant (R06-2002-004-01001-0) from the Basic Research Program of the Korea Science \& Engineering Foundation.

\section{References}

1. Harborne. J. B. : Williams, C. A. Nat. Prod Rep. 2001, 18, 310.

2. Schutz. B. A.: Wright. A. D.: Rali. T.: Sticher. O. Plytochemisty $1995,+0.1273$

3. Lin. Y. M.: Zhou. Y.: Flavin. M. T.: Zhou. L. M. Bioorgatic \& Medicinal Chentistry 2002. 10,2795.

4. (a) Ballesteros, J. F.: Sanz, M. J.: Utheda. A.: Miranda, M. A.; Iborra. S.: Paya, M.; Alearaz, M. J. J. 1 fed Chem. 1995. 38, 2794 (b) Wu. E. S. C.: Loch IL. I.: Toder. B. H.: Borrelli. A. R.: Gawlak. D.: Radow. L A.: Gensmantel. N. P. J. Hed. Chem. 1992. 35.3519 .

5. Jang, D. S.: Cuendet. M.: Hawthorne, M: Kardono, L. B. S.; Kawanishi, K.: Fong, H. H. S.: Mehta. R. G.: Pezzuto, J. M.; Kinghorn. A. D. Phytochemistry $\mathbf{2 0 0 2}, 61,867$.

6. (a) Olszanecki. R.: Gebska. A.: Kozlovski. V. I.: Gryglewski. R. J European J. Phamacology 2001. 25. 153. (b) Chi. Y. S.: Lim. H.: Park. H.: Kim. H. P. Biochentical Pharnacologe 2003. 66. 1271 (c) Dao. T. T; Chi. Y. S; Kim, J; Kim, H. P.; Park, H. Akh. Pharm. Res. 2003, 26. 345.

7. Sartor. L.: Pezzato. E.: Dell'Aica. I.: Caniato, R:; Biggin, S.; Garbisa. S. Biochenical Phamacolog, 2002. 6+. 229.

8. Rotelli. A. E.: Gurardia. I.: Juarez. A. O.: Rocha. N. E.: Pelzer. L. E. Pharntacological Researh 2003. +8.601 .

9. Wakabayashi. I.: Yasui, K. Eur J. Phamacol 2000. $406,477$.

10. Chi. Y. S.; Cheon, B. S.; Kim, H. P. Biochemical Phamacology 2001. 61, 1195 .

11. Chen. Y. C.: Shen. S. C.: Chen. L. G.: Lww. I. J.: Yang. L. L. Biochemical Phamacolog 2001.61. 1417.

12. (a) Li. L.: Wang. H. K.: Kuo. S. C.: Wu. T. S.: Lednicer. D.: Lin1. C. M. Hamel. E.; Lee. K. H. J. Med. Chent 1994, 37, 1126. (b) Li. L.: Wang. H. K.; Kuo. S. C.: Wu. T. S.: Mauger, A.; Lin, C. M: Hamel. E.: Lee, K. H. J. Ifed. Chem 1994, 37.3400.

13. Kulo. S. C.: Lee. H. Z.: Juang. T. P.: Linl. Y. T.: Wu. T. S.: Chang. J. J.: Lednicer. D.: Paull. K. D.: Lin. C. M.: Hamel. E.: Lee. K. H. J. Med. Chent 1993. 36. 1146.

14. Watterson. S. H.; Carlsen, M:- Dhar. T. G. M:- Shen, Z.; Pitts, W. J; Gu. H. H. Norris, D.: Chorba, J:- Chen. P; Cheny. D; Witmer. M:F Fleener. C. A.: Rouleau, K.: Townsend. R.; Hollenbaugh, D. L.: Iwanowicz. E. T. Bioorganic \& Hedicinal Chemisty Letters 2003. 13. 543 .

15. Xia. Y: Yang. Z. Y.: Xia. P.: Bastow: K. F.: Nakanishi. P. N.: Hamel. E.; Brossi. A.: Lee. K. H. Bioorganic \& Medicinal Chemistry Letters 2003. 13, 2891.

16. Cho. Y. S.: Kang. K. H. Cha, J. H.: Choi. K. I; Pae. A. N.: Koh. H. Y.: Chang. M. H. Bull. Korean Chem. Soc. 2002. 23(9). 1285. 